\title{
Are Accurate Equation of State Parameters Important in Richtmyer-, Meshkov Instabilities?
}

\author{
Lawrence D. Cloutman
}

August 1999

U.S. Department of Energy

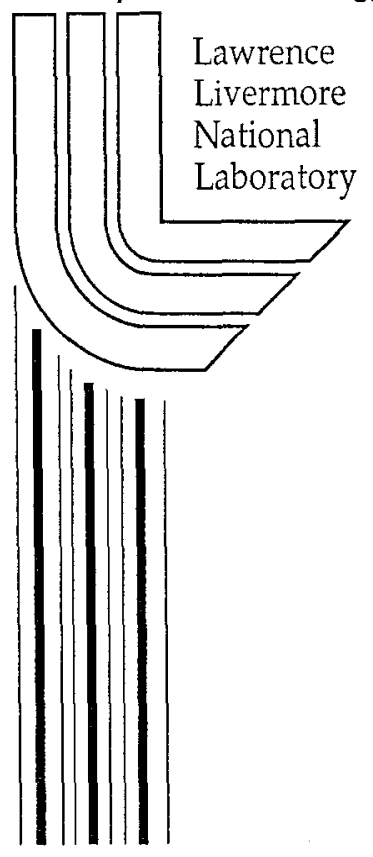




\section{DISCLAIMER}

This document was prepared as an account of work sponsored by an agency of the United States Government. Neither the United States Government nor the University of California nor any of their employees, makes any warranty, express or implied, or assumes any legal liability or responsibility for the accuracy, completeness, or usefulness of any information, apparatus, product, or process disclosed, or represents that its use would not infringe privately owned rights. Reference herein to any specific commercial product, process, or service by trade name, trademark, manufacturer, or otherwise, does not necessarily constitute or imply its endorsement, recommendation, or favoring by the United States Government or the University of California. The views and opinions of authors expressed herein do not necessarily state or reflect those of the United States Government or the University of California, and shall not be used for advertising or product endorsement purposes.

Work performed under the auspices of the U. S. Department of Energy by the University of Californita Lawrence Livermore National Laboratory under Contract W-7405-Eng-48.

This report has been reproduced directly from the best available copy.

Available to DOE and DOE contractors from the Office of Scientific and Technical Information

P.O. Box 62, Oak Ridge, TN 37831

Prices available from (423) 576-8401

http://apollo.osti.gov/bridge/

Available to the public from the National Technical Information Service

U.S. Department of Commerce 5285 Port Royal Rd., Springfield, VA 22161

http://www.ntis.gov/

OR

Lawrence Livermore National Laboratory

Technical Information Department's Digital Library

http://www.llnl.gov/tid/Library.html 
Lawrence Livermore National Laboratory Report

August 1999

ARE ACCURATE EQUATION OF STATE PARAMETERS
IMPORTANT IN RICHTMYER-MESHKOV INSTABILITIES?

Lawrence D. Cloutman

\begin{abstract}
The Richtmyer-Meshkov instability is a classical fluid dynamical instability that has been extensively studied to help understand turbulent mixing. A recent numerical simulation of a shock tube experiment with an an air- $\mathrm{SF}_{6}$ interface and a weak shock (Mach 1.2) used the ideal gas equation of state for air and an artifically low temperature as a surrogate for the correct $\mathrm{SF}_{6}$ gas physics. We have run a similar problem with both the correct gas physics and three versions of the air surrogate to understand the errors thercby introduccd. We find that for the weakly driven single-mode case considcred here, the instability amplitude is not affected, the interface location is affected only slightly, but the thermodynamic states are quite different. This result is not surprising because the flow far from the shock waves is essentially incompressible.
\end{abstract}




\section{Introduction}

One of the classical, archetypal fluid dynamical instabilities is the Richtmyer-Meshkov instability. In this instability, a shock wave impinges on a contact surface between two fluid layers with different densities. Vorticity induced at the contact surface by baroclinic torques during the shock passage leads to fluid motions that persist due to inertia. This instability has been extensively studied experimentally in shock tubes, and numerous numerical simulations have been performed with a wide variety of computational fluid dynamics programs (for example, see reference [1] and references therein).

Soon-to-be-published calculations [2] attempt to model an experiment [3] in which a shock travels from air into sulfur hexafluoride at modest Mach numbers. Unfortunately, the computer program used is restricted to a single fluid, so both fluids were approximated as a single ideal gas with a ratio of specific heats $\gamma=1.3$. The density difference of a factor of 5 was imposed by a temperature difference of the same factor rather than by using different molecular weights. The present study uses similar calculations with both correct and incorrect gas physics to determine the magnitude of the errors introduced by using the unphysical equation of state parameters.

The present calculations were performed with the COYOTE program [4], which solves the two-dimensional multicomponent Navier-Stokes equations. The problem solved is a simulation of the shock tube experiment by Benjamin [5], who also used air and $\mathrm{SF}_{6}$ with a Mach 1.24 shock. This experiment has been studied previously with a different computer program [1]. In the present study, we computed cases with four models for the $\mathrm{SF}_{6}$ gas physics. All four assumed a constant- $\gamma$ ideal gas. In the first case, we took the correct values of $\gamma=1.0935$ and a molecular weight of 146.0544 for the $\mathrm{SF}_{6}$. In the second case, we changed the $\gamma$ to 1.4 but retained the correct molecular weight. In the third case, we approximated the $\mathrm{SF}_{6}$ by cold air with $\gamma=1.4$ and molecular weight 28.9256 . The fourth case uses the equation of state of reference [2], which is the same as our third case but with $\gamma=1.3$ for both fluids.

Section 2 summarizes the governing equations. Section 3 presents numerical examples from COYOTE. Section 4 discusses the implications of these results. 


\section{Governing Equations}

The simulations were performed with the COYOTE computational fluid dynamics program [4], which is based on the full transient multicomponent Navier-Stokes equations in two dimensions. The model includes real-gas equations of state, arbitrary chemical kinetics, transport coefficients from a Lennard-Jones model, a simple radiative heat loss model, and mass diffusion based on the full Stefan-Maxwell equations. Chemistry and radiation are omitted from the present calculations.

Mass conservation is expressed by the continuity equation for each species $\alpha$ :

$$
\frac{\partial \rho_{\alpha}}{\partial t}+\nabla \cdot\left(\rho_{\alpha} \mathbf{u}\right)=-\nabla \cdot \mathbf{J}_{\alpha}
$$

where $\rho_{\alpha}$ is the density of species $\alpha, \mathbf{u}$ is the fluid velocity, and $\mathbf{J}_{\alpha}$ is the diffusional mass flux of species $\alpha$. The momentum equation is

$$
\frac{\partial(\rho \mathbf{u})}{\partial t}+\nabla \cdot(\rho \mathbf{u u})=-\nabla P+\nabla \cdot \mathcal{T}
$$

where $\rho$ is the total density and $P$ is the pressure. Because we consider only fluids, we assume that the viscous stress tensor is

$$
\mathcal{T}=\mu\left[\nabla \mathbf{u}+(\nabla \mathbf{u})^{\mathrm{T}}\right]-\frac{2 \mu}{3}(\nabla \cdot \mathbf{u}) \mathcal{U}
$$

where $\mu$ is the coefficient of viscosity, and $\mathcal{U}$ is the unit tensor. We choose the thermal internal energy equation to express energy conservation:

$$
\frac{\partial(\rho I)}{\partial t}+\nabla \cdot(\rho I \mathbf{u})=-P \nabla \cdot \mathbf{u}+\mathcal{T}: \nabla \mathbf{u}+\nabla \cdot\left[K \nabla T-\sum_{\alpha} h_{\alpha}(T) \mathbf{J}_{\alpha}\right],
$$

where $I$ is the specific thermal internal energy, $K$ is the mixture thermal conductivity, and $h_{\alpha}$ is the specific enthalpy of species $\alpha$.

The thermal equation of state assumes $P$ is given by the sum of the partial pressures of an ideal gas for each species. The JANAF tables $[6,7,8]$ provide a homogeneous set of thermochemical data for a large collection of materials, and these tables normally are used to supply the specific enthalpy for each species of interest. However, the limited range of temperatures encountered in the present study allows us to use a constant specific heat at constant volume for the caloric equation of statc.

In the present study, we use the Lennard-Jones model to estimate the transport coefficients [9]. This model provides a viscosity (in cgs units; to get SI units, multiply the 
cgs viscosity by 0.1 ) for each species,

$$
\mu_{\alpha}=\frac{5}{16}\left(\frac{m_{\mathrm{H}} k_{\mathrm{B}}}{\pi}\right)^{1 / 2} \frac{\left(M_{\alpha} T\right)^{1 / 2}}{\sigma_{\alpha}^{2} \Omega_{\nu}}=2.6693 \times 10^{-5} \frac{\left(M_{\alpha} T\right)^{1 / 2}}{\sigma_{\alpha}^{2} \Omega_{\nu}}
$$

where $M_{\alpha}$ is the molecular weight, $m_{\mathrm{H}}$ is the mass of one atomic mass unit in grams, $k_{\mathrm{B}}$ is the Boltzmann constant, $\sigma_{\alpha}$ is the collision diameter (in $\AA$ ), and $\Omega_{\nu}$ is the collision integral approximated by

$$
\Omega_{\nu}=1.147\left(T / T_{e \alpha}\right)^{-0.145}+\left(T / T_{e \alpha}+0.5\right)^{-2}
$$

where $T / T_{e \alpha}=T k_{\mathrm{B}} / \epsilon_{\alpha}$ is the reduced temperature and $\epsilon_{\alpha}$ is the Lennard-Jones potential well depth $[10,11]$. Equation 6 is accurate to a few percent for Monchick and Mason's [12] $\delta<$ 0.5 at low temperatures, and it becomes valid for larger values of $\delta$ at higher temperatures.

Once the species viscosities have been calculated, they must be combined to provide the viscosity of the fluid mixture. We adopt Wilke' law [13] (see also Bird et al. [14]). For $N$ species,

$$
\mu=\sum_{\alpha=1}^{N} \frac{X_{\alpha} \mu_{\alpha}}{\sum_{\beta=1}^{N} X_{\beta} \Phi_{\alpha \beta}}
$$

where $X_{\alpha}$ is the mole fraction of species $\alpha$ and where

$$
\Phi_{\alpha \beta}=8^{-1 / 2}\left(1+\frac{M_{\alpha}}{M_{\beta}}\right)^{-1 / 2}\left[1+\left(\frac{\mu_{\alpha}}{\mu_{\beta}}\right)^{1 / 2}\left(\frac{M_{\beta}}{M_{\alpha}}\right)^{1 / 4}\right]^{2}
$$

In the original COYOTE program, $K$ was calculated from the mixture viscosity $\mu$ and a constant mixture Prandtl number. This capability has been expanded to allow calculation of the conductivity based on the local composition and temperature, just as was done for the viscosity. Following the procedure of Hayashi and Hishida [10], we calculate the conductivity for each species from the species viscosities using the Eucken correction, which is discussed also by Ferziger and Kaper [15] and by Hirschfelder, Curtiss, and Bird [16]:

$$
K_{\alpha}=0.25\left(9 \gamma_{\alpha}-5\right) \mu_{\alpha} C_{\mathrm{v} \alpha}
$$

where $C_{\mathrm{v} \alpha}=R / M_{\alpha}\left(\gamma_{\alpha}-1\right)$ is the specific heat at constant volume, $R$ is the universal gas constant, and $\gamma_{\alpha}$ is the ratio of specific heats. The mixture rule that we use for conductivities is due to Mathur et al. [17] and is recommended by Kee, et al. [18]:

$$
K=\frac{1}{2}\left[\sum_{\alpha=1}^{N} X_{\alpha} K_{\alpha}+\left(\sum_{\alpha=1}^{N} X_{\alpha} / K_{\alpha}\right)^{1}\right] \text {. }
$$


In general, calculation of the diffusional mass fluxes is a complicated task (for example, [14], [16], and [19]). Because molecular mass diffusion effects are small in the present problem, we simply use Fick's law,

$$
\mathbf{J}_{\alpha}=-\rho D_{\alpha} \nabla\left(\rho_{\alpha} / \rho\right)
$$

where $D_{\alpha}$ is the species diffusivity. We use the same value of $D_{\alpha}$ for all species, and this value is given as the kinematic viscosity of the mixture divided by a constant mixture Schmidt number, which we took to be 0.9 . This simple model has the advantages that it is computationally inexpensive and the species mass fluxes properly add up to zero when summed over species.

\section{Numerical Simulations}

Table 1 gives the COYOTE input file for the problem with the correct gas physics for $\mathrm{SF}_{6}$. We assume the fluids are ideal gases with constant ratios of specific heats that are computed from the input values of $\gamma_{\alpha}$ and $M_{\alpha}$. The computational grid is $34.0 \mathrm{~cm}$ long. The left $10.0 \mathrm{~cm}$ is covered by 200 uniform zones and is the part of the grid where the instability occurs. The right $24.0 \mathrm{~cm}$ is covered by a stretched grid of 140 zones and provides a reservoir where waves reflected from the contact surface may safely propagate away from the region of interest. The grid is $3.75 \mathrm{~cm}$ high and is spanned by 75 uniform zones. This height is one wavelength of the initial single-mode perturbation of the air-SF $\mathrm{S}_{6}$ contact surface. The intial peak-to-peak amplitude of the perturbation is $0.48 \mathrm{~cm}$. Temperature in the unshocked fluid is $287.7 \mathrm{~K}$. The pressure in the unshocked fluid was assumed to be $1.0 \mathrm{~atm}$, although it actually may have been closer to $0.8 \mathrm{~atm}$ in the experiment due to the $2100 \mathrm{~m}$ elevation of Los Alamos. However, the results of interest here are independent of this baseline pressure [1].

Figure 1 shows the density in the left $10.178 \mathrm{~cm}$ of the grid at $t=0.0 \mathrm{~ms}$. There are nine contours lines, and each pair of contours is separated by 10 percent of the difference between the maximum and minimum values of the density in the plotted region. The single contour at the far right is the Mach 1.24 shock wave, which is propagating to the left. The closely spaced contours to the left of the shock are the initial interface (that is, the contact surface), which has its average location $7.5 \mathrm{~cm}$ from the left boundary.

Figure 2 shows the density at $t=0.6 \mathrm{~ms}$. This problem was run with convective fluxes that are a mixture of 0.2 donor cell and 0.8 tensor viscosity differencing and with the correct 
gas parameters. The vertical contcurs at the left are the shock, which has been reflected off the left wall of the grid. The curved contours are the air-SF 6 contact surface. The code calculates the problem as a multicomponent fluid without an explicit interface treatment. The broadening of the contact surface when compared to Figure 1 is due to the diffusive truncation errors in the advective fluxes, with only a minor contribution from molecular diffusion. This numerical diffusion is needed to keep dispersive truncation errors under control since this code has no advective flux limiters. COYOTE was designed primarily for subsonic flows and deflagration waves, and experience shows that flux limiters are of limited utility under those conditions.

Figure 3 is the same as Figure 2 except the convective fluxes are computed with donor cell differencing. The numerical diffusion is quite a bit larger in this case, but the location, shape, and peak-to-peak amplitude of the contact surface is nearly the same as with the more accurate method used in Figure 2. Overlaying the plots, one can see that the four rightmost contours in Figure 2 nearly coincide with the $c$ and d contours of Figure 3 . This result suggests that numerical diffusion is more of an aesthetic problem than an accuracy issue for our present purposes.

The density contours shown in Figure 4 are from a calculation that is the same as that shown in Figure 2, except we have used twice as many zones in each direction. The only difference is in the thickness of the shock and contact surface, as expected.

Figure 5 is the same as Figure 2, except we used $\gamma=1.4$ instead of $\gamma=1.0935$ in the $\mathrm{SF}_{6}$. The biggest difference is that the shock has traveled approximately $1 \mathrm{~cm}$ farther to the right in Figure 5. The contact surface has moved only about $2 \mathrm{~mm}$ farther to the left, which is a little surprising given the significant stiffening of the gas by the larger $\gamma$ in Figure 5. However, the original shock is not very strong, and consequently not only is the degree of compression modest in the singly-shocked gas, it is not terribly sensitive to the details of the equation of state. The amplitude of the instability is virtually unaffected by the change in the equation of state, which is not surprising given that the baroclinic torques that drive the instability depend only on the density and pressure gradients during shock passage, and these are essentially the same in all four cases.

Figure 6 shows the density contours for the case where the $\mathrm{SF}_{6}$ has been replaced by air with the temperature lowered by a factor of approximately 5 to get the density of $\mathrm{SF}_{6}$ while maintaining the pressure of one atmosphere. There is no significant difference between this plot and Figure 5 as far as the amplitude of the instability and the locations of the shock 
and contact surface are concerned.

This is all well and good, but the run described in reference [2] that led to this study used a one-component model with the correct molecular weight for air, but used $\gamma=1.3$ as "a compromise" between the correct values in the two fluids. This unfortunatc choice causes a problem at the start because the experimental papers give no information about the original shock except for its Mach number in air. We have to assume that the unshocked air is at room temperature and pressure. By changing the $\gamma$ of the air from 1.4 to 1.3 , we must also change the post-shock conditions to hold the Mach number fixed. The velocity increases from $1.229 \times 10^{4}$ to $1.321 \times 10^{4} \mathrm{~cm} / \mathrm{s}$, the singly-shocked air density increases to $1.8078 \times 10^{-3}$ from $1.7285 \times 10^{-3} \mathrm{~g} / \mathrm{cm}^{3}$, and the temperature decreases to 324.0 from 331.8 $K$. Since the unshocked density is $1.2250 \times 10^{-3}$, the density jump across the shock increases by 16 percent. Figure 7 shows the density contours for this case at $t=0.6 \mathrm{~ms}$. If we ignore the $\mathrm{g}$ contour in Figure 6 and translate the contours $3 \mathrm{~mm}$ to the right in Figure 7 , then the contours coincide. In other words, the instability amplitude is still independent of the choice of $\gamma$, but the translation speed is slightly higher for the lower $\gamma$.

The remaining figures show the distributions of the hydrodynamical variables along the centerline of the grid for the four equations of state. The calculations shown here were done with donor cell differencing to suppress the dispersive truncation errors without altering the jump conditions.

Figure 8 shows the distributions of density for all four equation of state models. The solid curve is for the correct equation of state for both gases. The high density fluid between 0 and $1 \mathrm{~cm}$ is $\mathrm{SF}_{6}$ that has been shocked twice. $\mathrm{SF}_{6}$ that has been shocked once lies between 1 and $3 \mathrm{~cm}$. The next $1.5 \mathrm{~cm}$ contain the numerically broadened contact surface. Note that this is the thickness of the contact surface at the rightmost peak in figure 2, not the amplitude of the instability. This much broadening of a contact surface that has moved approximately 4 cm from its original position is why we normally avoid using donor cell differencing. However, donor cell differencing will produce the correct jump conditions without dispersively driven oscillations, which would only obscure the features of interest here. Between 5 and $10 \mathrm{~cm}$ we have air that was shocked first by the incoming shock, and then reshocked by the shock wave reflected from the contact surface. As noted in the discussion of the contour plots, the contact surface location varies only about $3 \mathrm{~mm}$ between the correct $\mathrm{SF}_{6}$ model and the three unphysical models, two of which are in perfect agreement. The densities of both the doubly and singly shocked $\mathrm{SF}_{6}$ show a dependence on $\gamma$, but not on the molecular weight 
for a fixed $\gamma$. Similarly, the location of the reflected shock depends on the value of $\gamma$ but not on the molecular weight.

Figure 9 shows the pressure distributions. Once again, the two unphysical $\gamma=1.4$ equations of state produce the same solutions, neither of which is quite the same as the solution with the correct parameters. In all cases, there is no pressure gradient across the contact surface. There is an interesting feature in the pressure in the incoming air, however. It has been reshocked by slightly different shocks reflected back to the right off the contact surface. The reflected shock strength depends slightly on the ratio of specific heats in the $\mathrm{SF}_{6}$, but not on the molecular weight.

Figure 10 shows the temperature distributions. Here we see big differences in both the singly and doubly shocked $\mathrm{SF}_{6}$. If you are doing a problem that requires accurate temperatures, such as chemical reaction rates or opacities, then clearly it is important to use the correct equation of state.

Figure 11 shows the internal encrgy distributions. The only point of interest is that unlike the temperature, the internal energies are the same for the two unphysical $\gamma=1.4$ equations of state. The internal energy for the singly-shocked air is the same also for the correct equation of state, but it is decidedly different for the "compromise" $\gamma=1.3$ solution.

Figure 12 shows the velocity component in the direction of the centerline. For the three $\gamma \neq 1.3$ solutions, the incoming air velocity does show a significant $\gamma$ dependence, and a very tiny molecular weight dependence. The bumps in the region around 3 to $4 \mathrm{~cm}$ are perturbations due to the two-dimensional motion that increases the amplitude of the instability in the contact surface.

Table 2 summarizes the instability (peak-to-peak) growth rates and translation speeds for the experiment and for two numerical solutions. One solution, Case 2, is from reference [1], in which the computed growth rate is twice the experimental growth rate. The other solution, Case 3, is taken from the run shown in figure 2. The numerical values were obtained by measuring graphical computer output and used the locations of the middle contour in the contact surface at two different times. This is the correct feature to measure in Case 3 because it minimizes errors in the growth rate due to numerical diffusion of the contact surface. The precision of these values is approximately $100 \mathrm{~cm} / \mathrm{s}$. The most notable feature is the significant reduction in the computed growth rate compared to Case 2. However, the COYOTE growth rate still is appreciably larger than the experimental value. The computed translation speeds are consistent with the experiment. 


\section{Conclusions}

We have completed numerical simulations of a Richtmyer-Meshkov instability experiment performed in a shock tube. The working fluids are air and $\mathrm{SF}_{6}$, and the shock speed is Mach 1.24. We used four different approximations to the ideal gas parameters for the $\mathrm{SF}_{6}$. This study demonstrates several things.

1. Numerical diffusion, while not negligible on the fairly coarse mesh used, does not seriously affect the parameters that we are most interested in. Since we are interested only in gross differential changes in the solution as we vary the equation of state parameters for the $\mathrm{SF}_{6}$, and since the one finer-mesh run showed no change in the parameters of interest, there is little point to running additional fine mesh cases.

2. The amplitude of the singly-shocked mixed layer seems to be independent of the molecular weights and ratios of specific heats. This is because the growth rate depends on the amount of vorticity generated by baroclinic torques during shock passage of the contact surface. If the pressure and density jumps are always the same, the vorticity generation with always be the same.

3. The contact surface translation speed and location are insensitive to the equation of state parameters. Perhaps this is a fortuitous consequence of the relatively weak shock wave.

4. The shock location just before the reshocking of the contact surface does depend slightly on the equation of state parameters, and the difference is expected to be more pronounced for strong shocks.

5. The post-shock thermodynamic conditions depend sensitively on the equation of state, even for this weak shock wave.

We note that these results may not carry over to cases with strong shock waves. Also, it remains to be demonstrated that the insensitivity to the details of the equation of state will carry over to the case of multimode contact surfaces, especially if there are wavelengths present that are comparable to the zone size. However, the equation of state errors in the calculations that inspired this study [2] should not be a significant source of error in the predicted growth rate of the mixed layer. 


\section{Acknowledgments}

I thank John Ramshaw for several helpful discussions. This work was performed under the auspices of the U.S. Department of Energy and the Lawrence Livermore National Laboratory under contract number W-7405-ENG-48.

\section{References}

[1] L. D. Cloutman and M. F. Wehner, "Numerical simulation of Richtmyer-Meshkov instabilities," Phys. Fluids A 4:1821 (1992).

[2] R. H. Cohen, W. P. Dannevik, A. Dimits, D. E. Eliason, A. Mirin, T. Peyser, O. Schilling, D. H. Porter, and P. R. Woodward, "Simulations of two-scale and doubleshocked Richtmeyer-Meshkov" (sic), Lawrence Livermore National Laboratory abstract. UCRL-JC-133258 Abs, 1999. To be presented at the 7th International Workshop on the Physics of Compressible Turbulent Mixing, St. Petersburg, Russia, July 5-9, 1999.

[3] M. Vetter and B. Sturtevant, "Experiments on the Richtmyer-Meshkov instability of an air/SF 6 interface," Shock Waves 4, 247 (1995).

[4] L. D. Cloutman, "COYOTE: A Computer Program for 2-D Reactive Flow Simulations," Lawrence Livermore National Laboratory report UCRL-ID-103611, 1990.

[5] R. F. Benjamin, "Experimental observations of shock stability and shock-induced turbulence," in $\Lambda$ dvances in Compressible Turbulent Mixing, CONF-8810234, editcd by W. P. Dannevik, A. C. Buckingham, and C. E. Leith (USGPO, Washington, 1992).

[6] D. R. Stull and II. Prophet, "JANAF Thermochemical Tables, 2nd ed.," U. S. Department of Commerce/National Bureau of Standards report NSRDS-NBS 37, 1971.

[7] M. W. Chase, J. L. Curnull, A. T. Hu, H. Prophet, A. N. Syverud, and L. C. Walker, "JANAF Thermochemical Table, 1974 Supplement," J. Phys. Chem. Ref. Data 3, 311 (1974).

[8] M. W. Chase, Jr., C. A. Davies, J. R. Downey, Jr., D. J. Frurip, M. A. McDonald, and A. N. Syverud, "JANAF Thermochemical Tables, Third Edition, Parts I and II," Supplement No. 1, J. Phys. Chem. Ref. Data 14 (1985). 
[9] L. D. Cloutman, "A Database of Selected Transport Coefficients for Combustion Studies," Lawrence Livermore National Laboratory report UCRL-ID-115050, 1993.

[10] A. K. Hayashi and M. Hishida, "Numerical Study on Pulsed Jet Combustion," in Fourth International Symposium on Computational Fluid Dynamics, Vol. III, University of California at Davis (1991), pp. 19-24.

[11] F. M. White, Viscous Fluid Flow (McGraw-Hill, New York, 1974).

[12] L. Monchick and E. A. Mason, "Transport properties of polar gases," J. Chem. Phys. 35, 1676 (1961).

[13] C. R. Wilke, "A Viscosity Equation for Gas Mixtures," J. Chem. Phys. 18, 517 (1950).

[14] R. B. Bird, W. E. Stewart, and E. N. Lightfoot, Transport Phenomena (Wiley, New York, 1960).

[15] J. H. Ferziger and H. G. Kaper, Mathematical Theory of Transport Processes in Gases (North-Holland, Amsterdam, 1972).

[16] J. O. Hirschfelder, C. F. Curtiss, and R. B. Bird, Molecular Theory of Gases and Liquids (Wiley, New York, 1964).

[17] S. Mathur, P. K. Tondon, and S. C. Saxena, "Thermal conductivity of binary, ternary, and quaternary mixtures of rare gases," Mol. Phys. 12, 569 (1967).

[18] R. J. Kee, G. Dixon-Lewis, J. Warnatz, M. E. Coltrin, and J. A. Miller, "A Fortran Computer Code Package for the Evaluation of Gas-Phase Multicomponent Transport Properties," Sandia National Laboratories report SAND86-8246, 1986; reprinted 1990.

[19] S. Chapman and T. G. Cowling, The Mathematical Theory of Non-Uniform Gases (Cambridge University Press, London, 1952). 
Table 1.

COYOTE Input File

Base Case Input (cgs units)

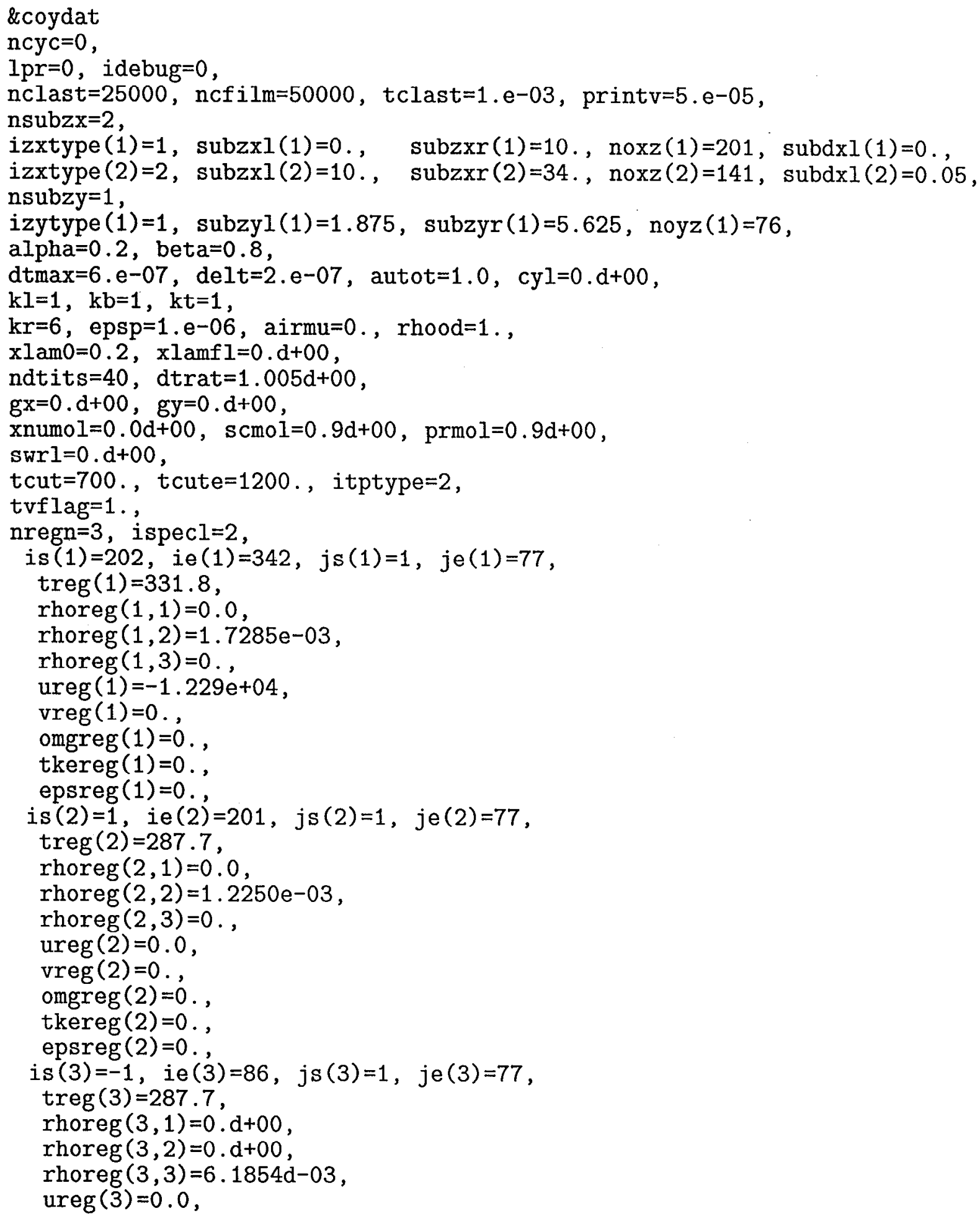




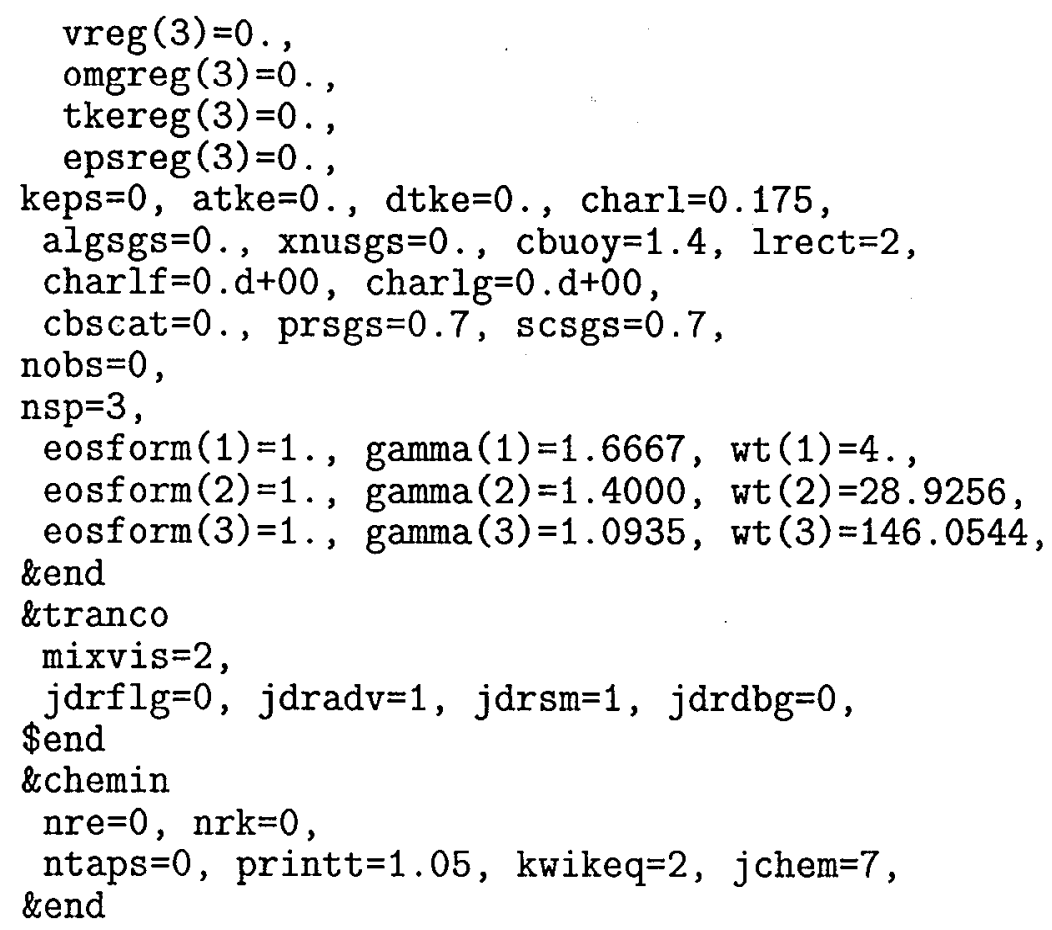

The gasdata file has 3 species, and we used the Lennard-Jones parameters:

Species

$1 \mathrm{He}$

$2 \mathrm{~N} 2$

3 SF 6 sigma (A)

2.576

3.621

5.252
epsilon/k_B

10.2

97.53 (used these for air)

207.7

Table 2. Growth Rates and Translation Velocities $(\mathrm{cm} / \mathrm{s})$

\begin{tabular}{llll}
\hline Case & EOS Parameters & Growth Rate & Translation Vel. \\
\hline 1 & Expt. [5] & 1580 & 8100 \\
2 & CW [1] & 3080 & 7800 \\
3 & Air-SF $_{6}$ (Figure 2) & 2420 & 7740 \\
\hline
\end{tabular}




\section{Density cycle $=0 \mathrm{t}=0.000000 \mathrm{D}+00 \mathrm{dt}=2.000000 \mathrm{D}-07$ $\max =6.185400 \mathrm{D}-03 \mathrm{~min}=1.225000 \mathrm{D}-03 \mathrm{dq}=4.960400 \mathrm{D}-04$}

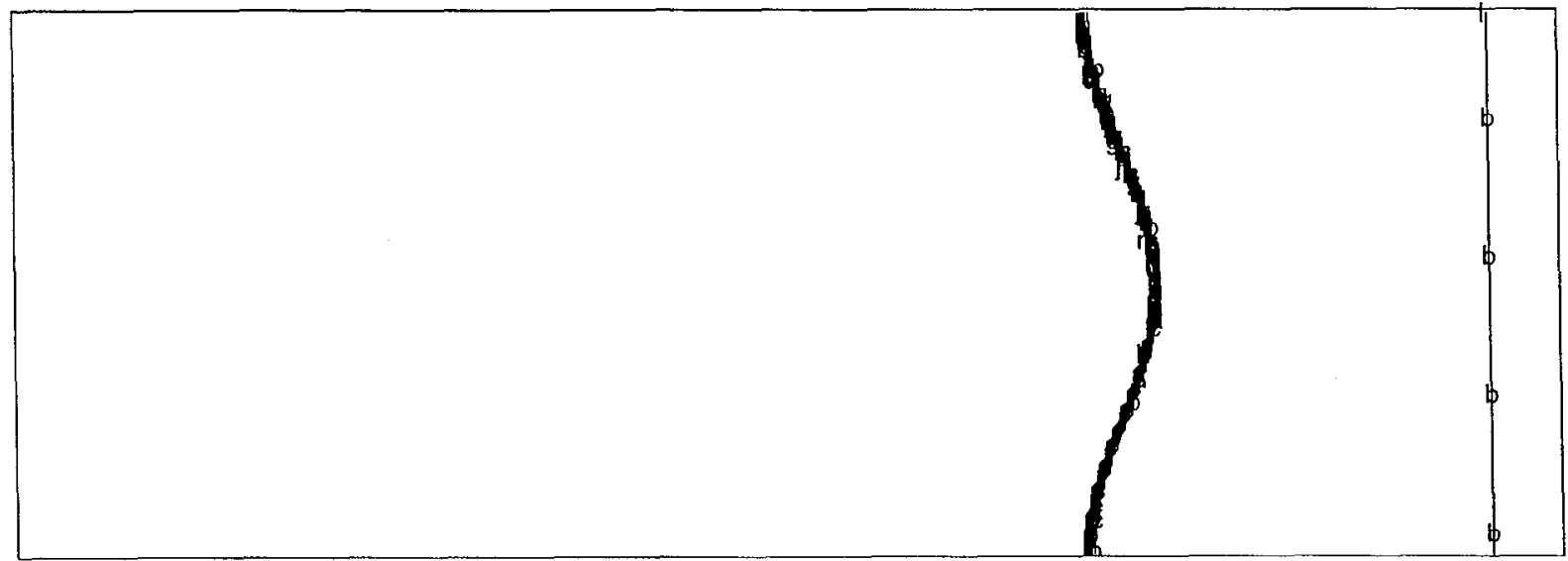

Figure 1: Density at $t-0.0 \mathrm{~ms}$ in the left $10.478 \mathrm{~cm}$ of the grid. The shock is seen at the far right, and it is moving to the left. The interface is $7.5 \mathrm{~cm}$ from the left. The first three figures use the correct gas physics for the $\mathrm{SF}_{6}$. 


\section{Density cycle $=1088 \mathrm{t}=6.002369 \mathrm{D}-04 \mathrm{dt}=6.000000 \mathrm{D}-07$ $\max =2.031632 \mathrm{D}-02 \mathrm{~min}=1.851387 \mathrm{D}-03 \mathrm{dq}=1.846493 \mathrm{D}-03$}

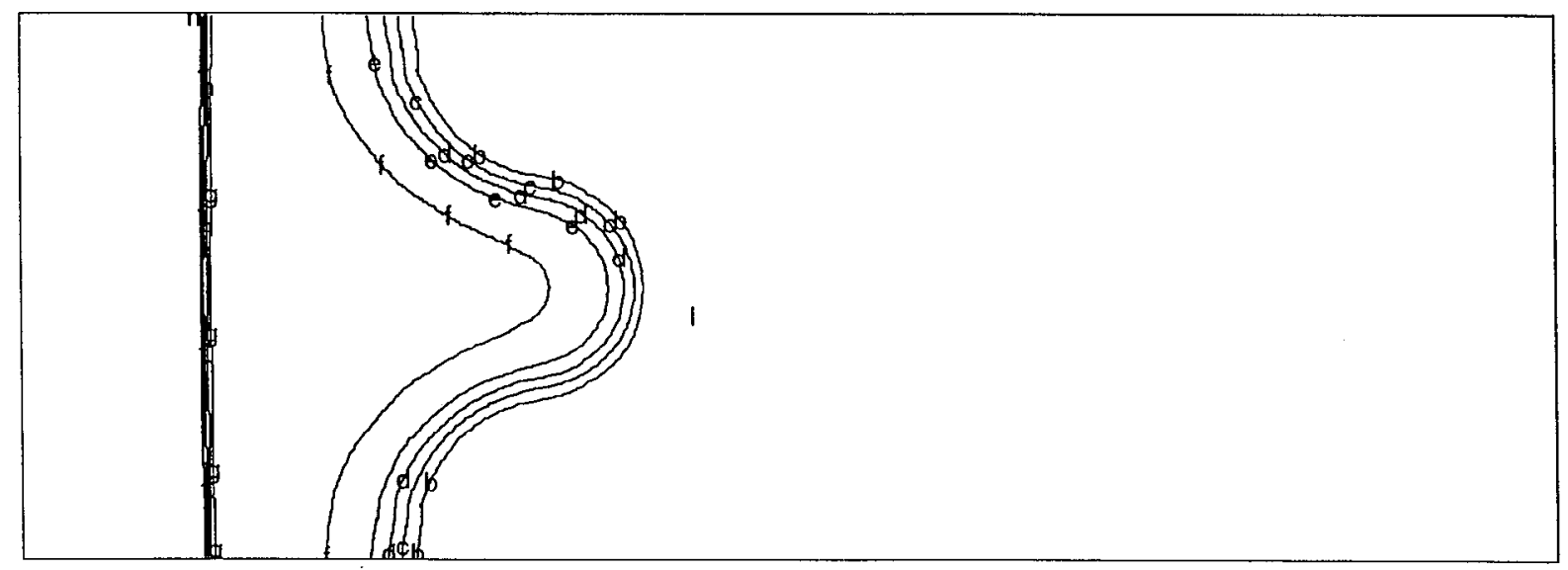

Figure 2: Density at $t=0.6 \mathrm{~ms}$. The vertical contours at the left are the shock wave, which has been reflected off the left boundary. The curved contours are the air-SF 6 contact surface. 


\section{Density cycle $=1088 \mathrm{t}=6.002369 \mathrm{D}-04 \mathrm{dt}=6.000000 \mathrm{D}-07$ $\max =1.973155 \mathrm{D}-02 \min =1.941900 \mathrm{D}-03 \mathrm{dq}=1.778965 \mathrm{D}-03$}

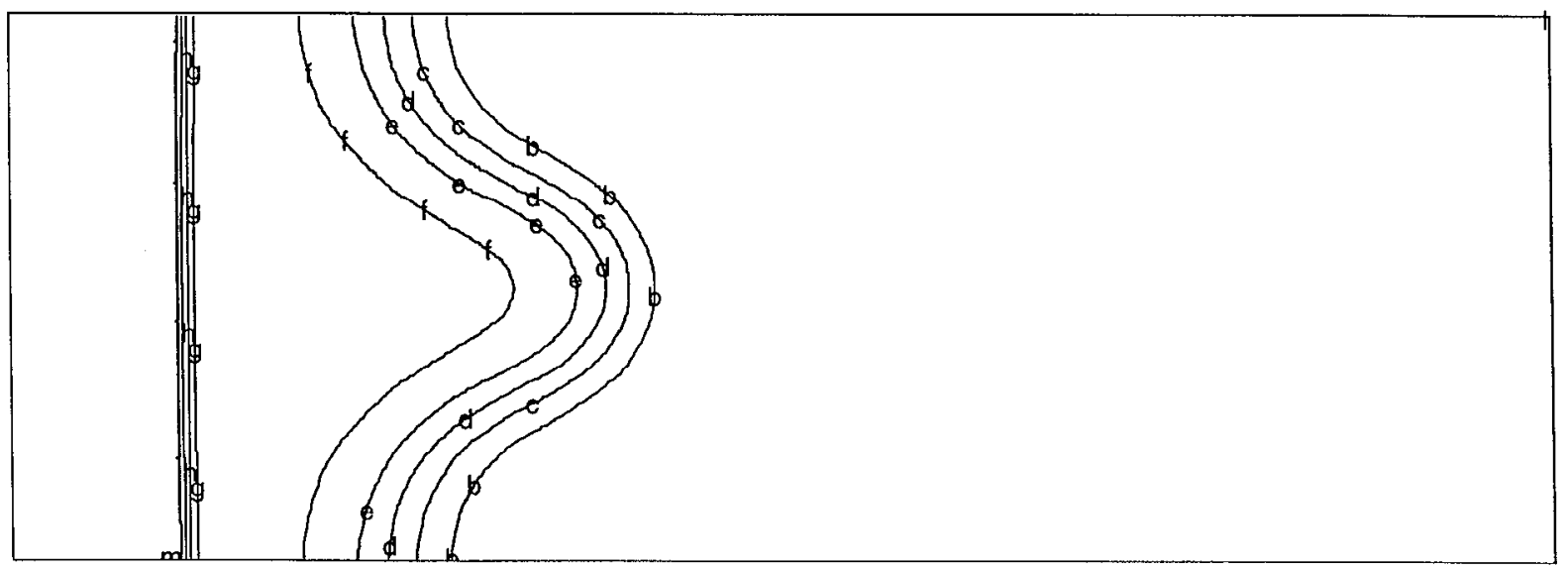

Figure 3: Density at $t=0.6 \mathrm{~ms}$, the same as the previous figure, except the run was made with pure donor cell differencing to test the effect of numerical diffusion on this problem. 


\section{Density cycle $=1373 \mathrm{t}=6.003324 \mathrm{D}-04 \mathrm{dt}=5.465480 \mathrm{D}-07$ $\max =1.999216 \mathrm{D}-02 \mathrm{~min}=1.887352 \mathrm{D}-03 \mathrm{dq}=1.810481 \mathrm{D}-03$}

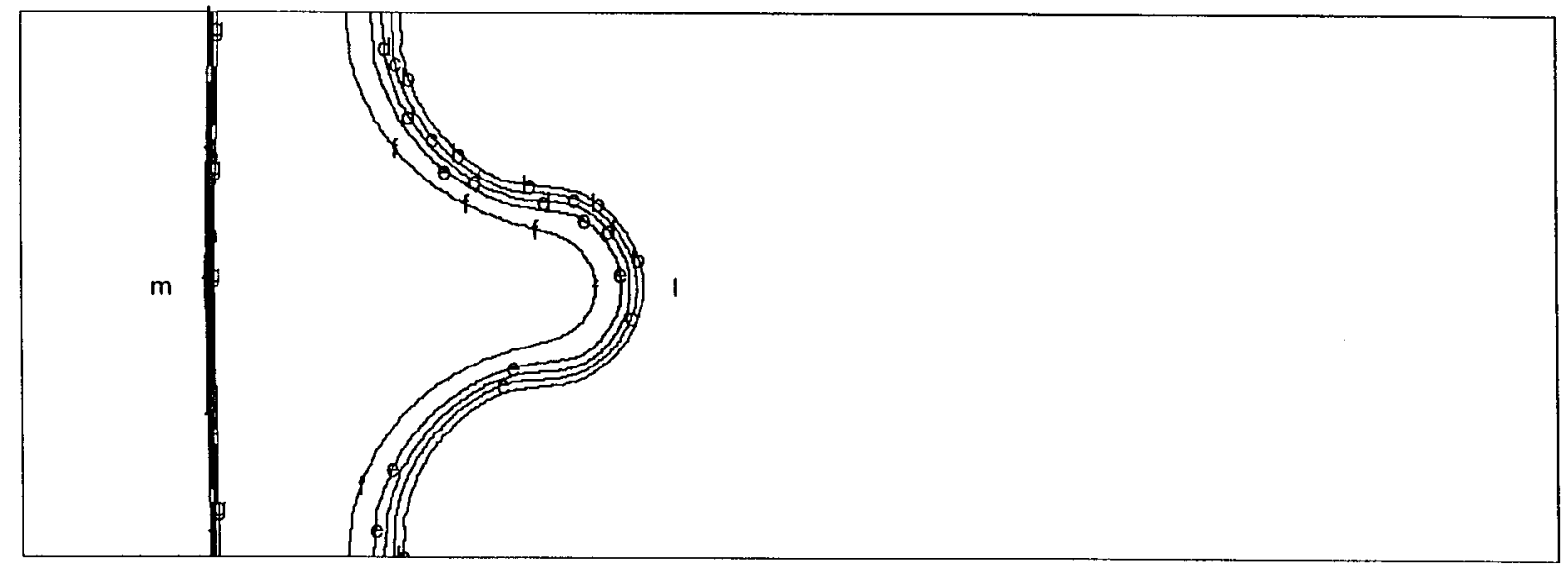

Figure 4: Density at $t=0.6 \mathrm{~ms}$ for the same case as shown in Figure 2, except this run has twice as many zones in each direction. 


\section{Density cycle $=1088 \mathrm{t}=6.002369 \mathrm{D}-04 \mathrm{dt}=6.000000 \mathrm{D}-07$ $\max =1.549932 \mathrm{D}-02 \min =1.941366 \mathrm{D}-03 \mathrm{dq}=1.355795 \mathrm{D}-03$}

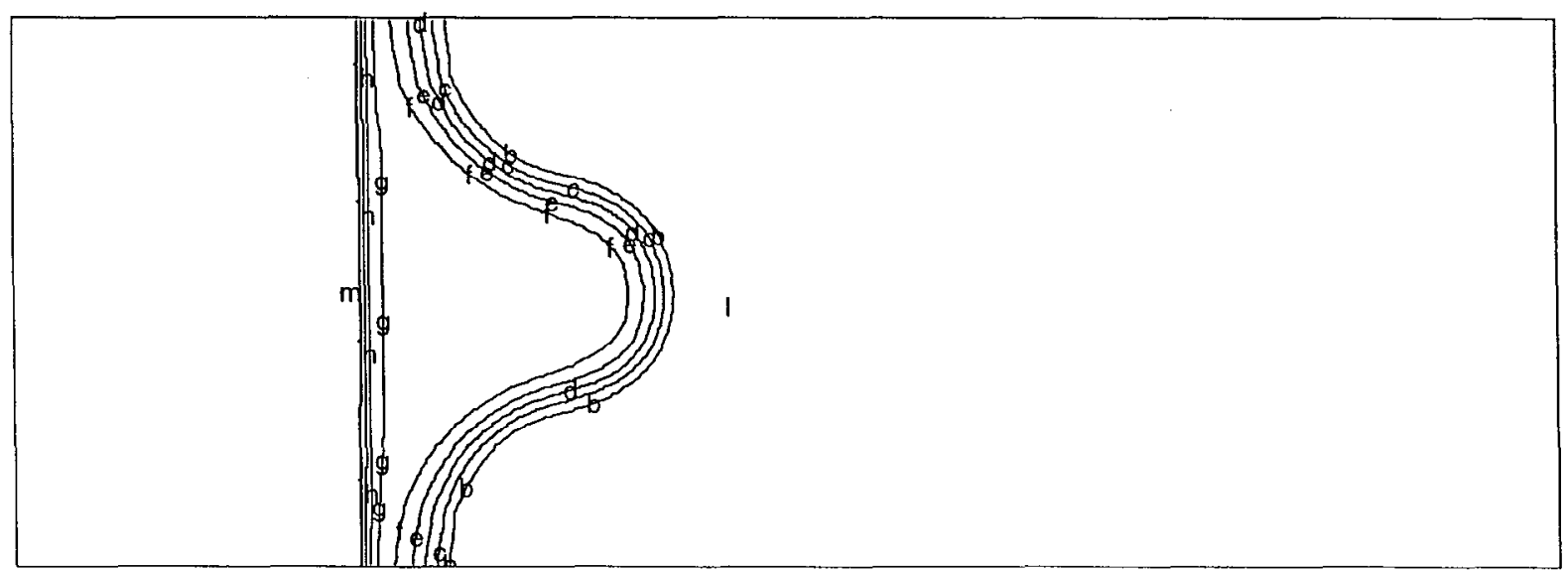

Figure 5: Density at $t=0.6 \mathrm{~ms}$ for the case with $\gamma=1.4$ and the correct molecular weight for the $\mathrm{SF}_{6}$. 


\section{Density cycle $=1088 \mathrm{t}=6.002369 \mathrm{D}-04 \mathrm{dt}=6.000000 \mathrm{D}-07$ $\max =1.553979 \mathrm{D}-02 \mathrm{~min}=1.427397 \mathrm{D}-03 \mathrm{dq}=1.411239 \mathrm{D}-03$}

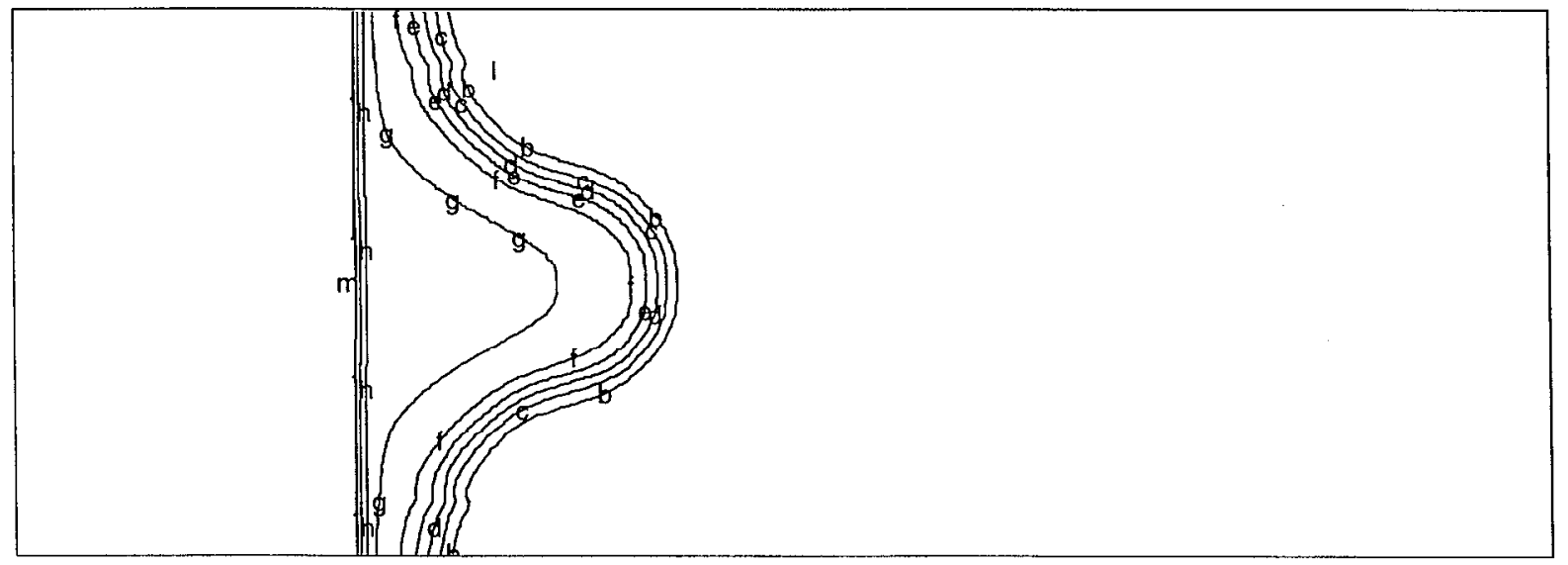

Figure 6: Density at $t=0.6 \mathrm{~ms}$ for the case where the $\mathrm{SF}_{6}$ is approximated by cold air with $\gamma=1.4$. 


\section{Density cycle $=1088 \mathrm{t}=6.002369 \mathrm{D}-04 \mathrm{dt}=6.000000 \mathrm{D}-07$ $\max =1.757307 \mathrm{D}-02 \min =1.507563 \mathrm{D}-03 \mathrm{dq}=1.606550 \mathrm{D}-03$}

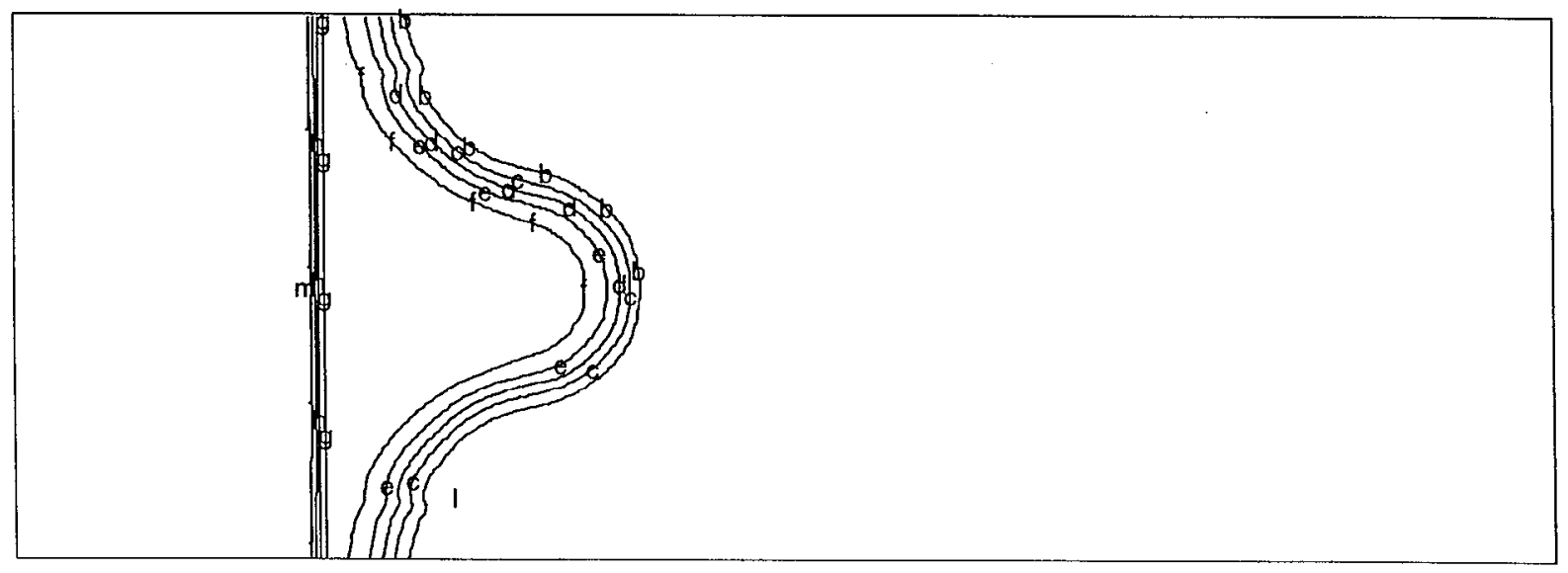

Figure 7: Density at $t=0.6 \mathrm{~ms}$ for the case where the $\mathrm{SF}_{6}$ is approximated by cold air. All regions of the fluid use $\gamma=1.3$, which affects the shock strength when we hold the Mach number fixed. 


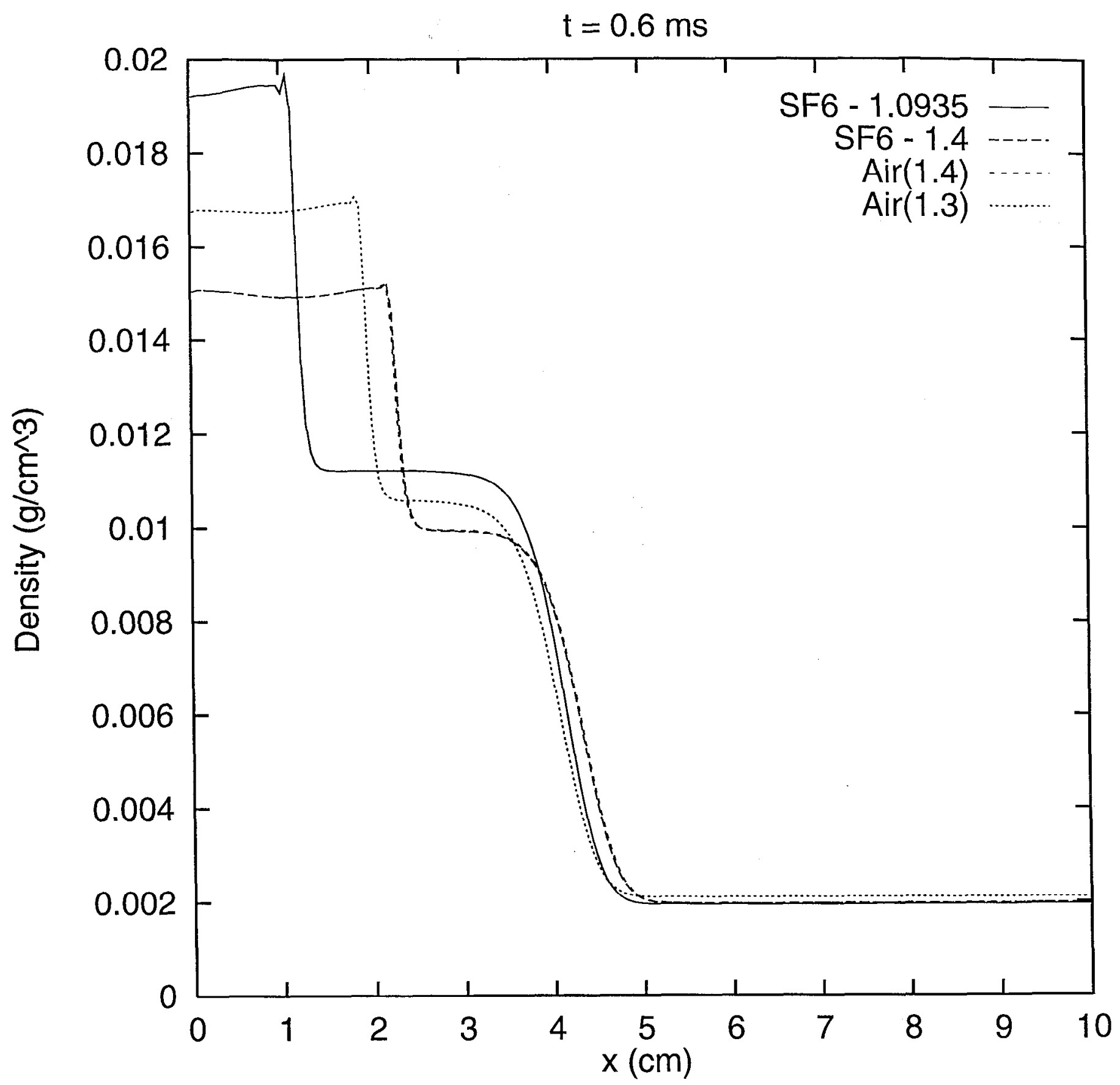

Figure 8: Density as a function of $x$ along the centerline of the grid at $t=0.6 \mathrm{~ms}$ for all four $\mathrm{SF}_{6}$ equation of state models. The solid line is for the correct $\mathrm{SF}_{6}$ equation of state, and the line with long dashes is for the correct molecular weights but $\gamma=1.4$. The line with short dashes is the casc with $\mathrm{SF}_{6}$ modeled as cold air, which has a different molecular weight and $\gamma=1.4$. The dotted line is the case with both gases represented by air with $\gamma=1.3$. The two dashed lines coincide in this plot. 


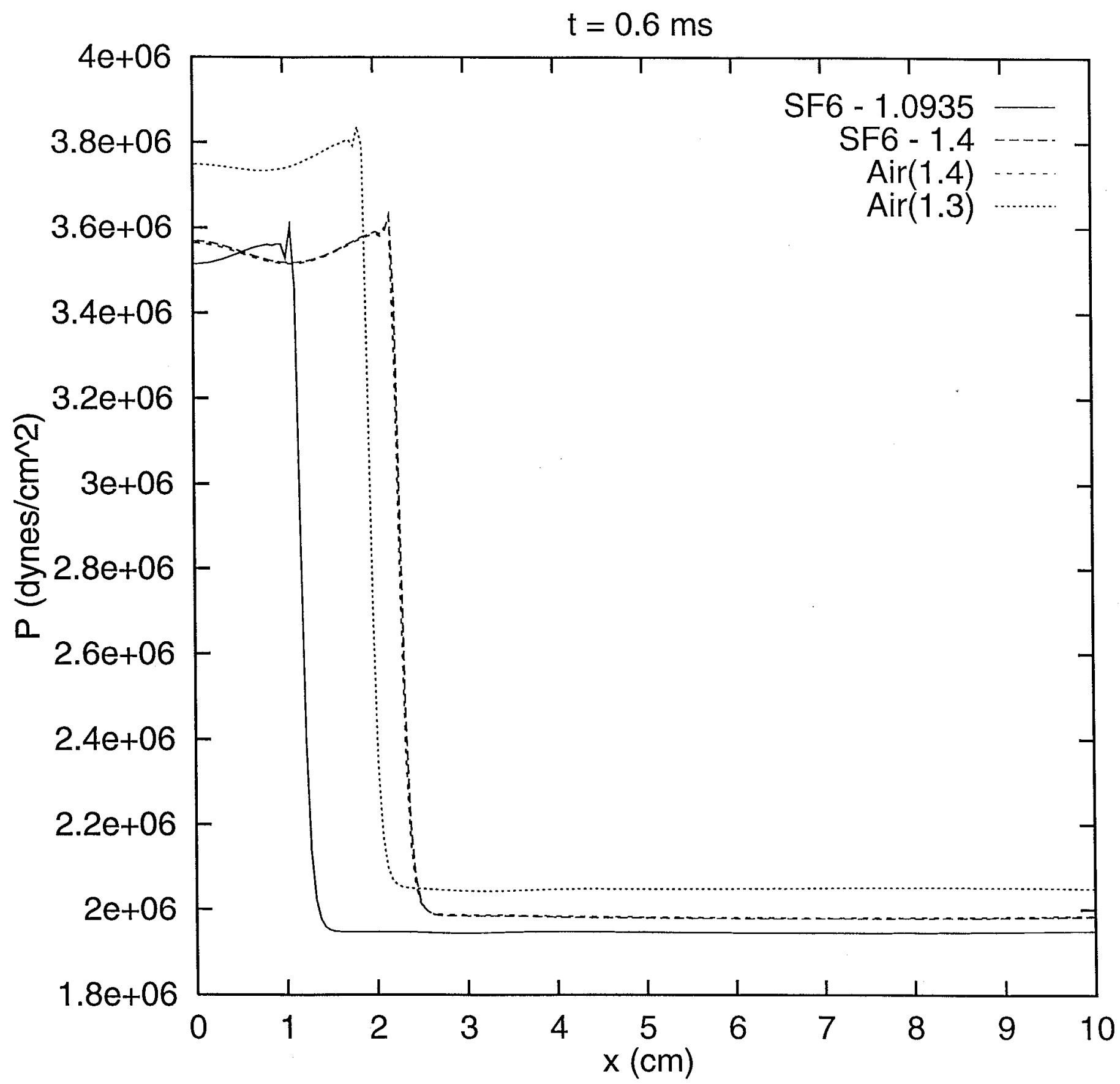

Figure 9: Pressure as a function of $x$ at $t=0.6 \mathrm{~ms}$ for all four cases. The two dashed lines coincide in this plot. 


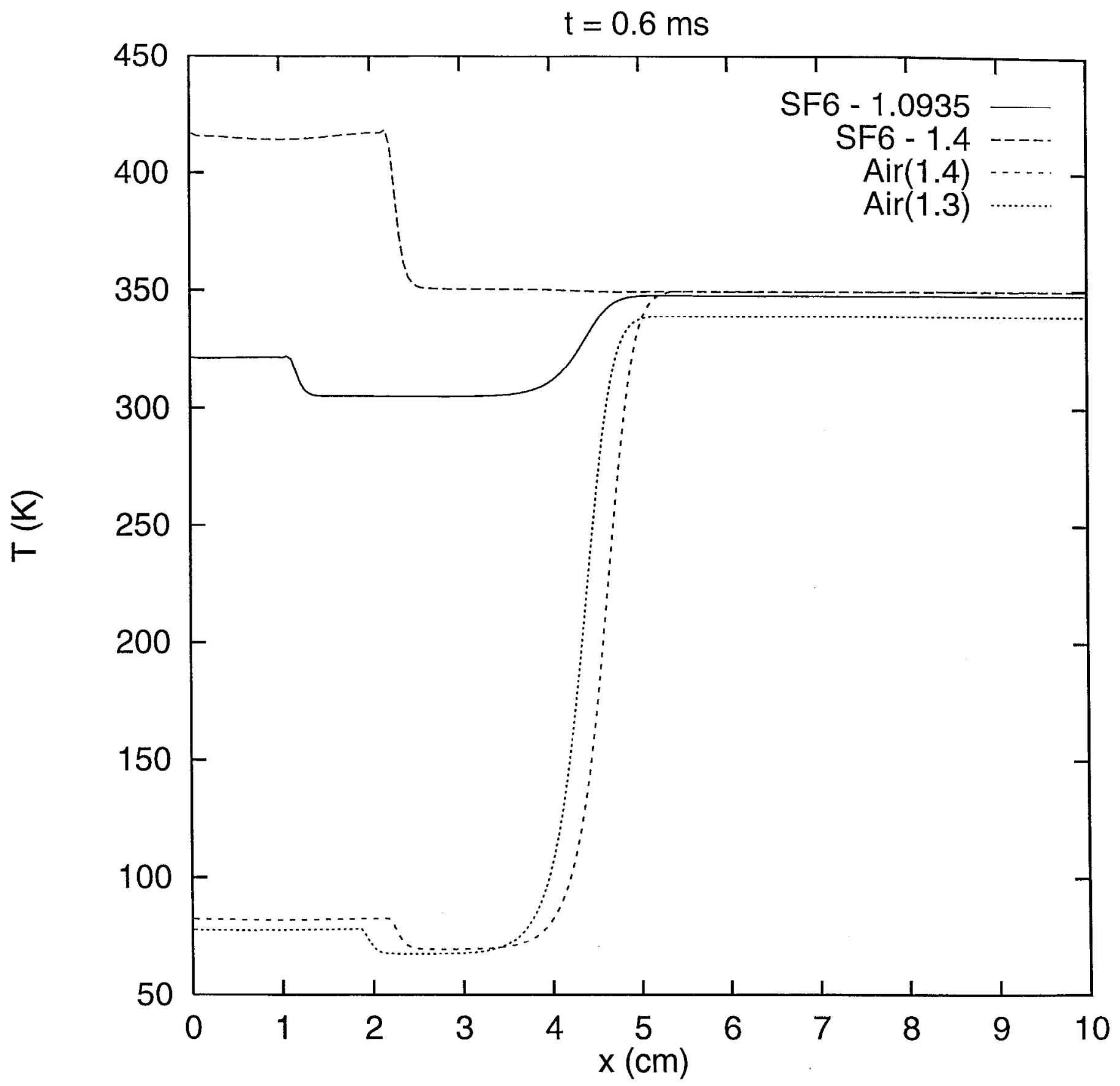

Figure 10: Temperature as a function of $x$ at $t=0.6 \mathrm{~ms}$ for all four cases. 


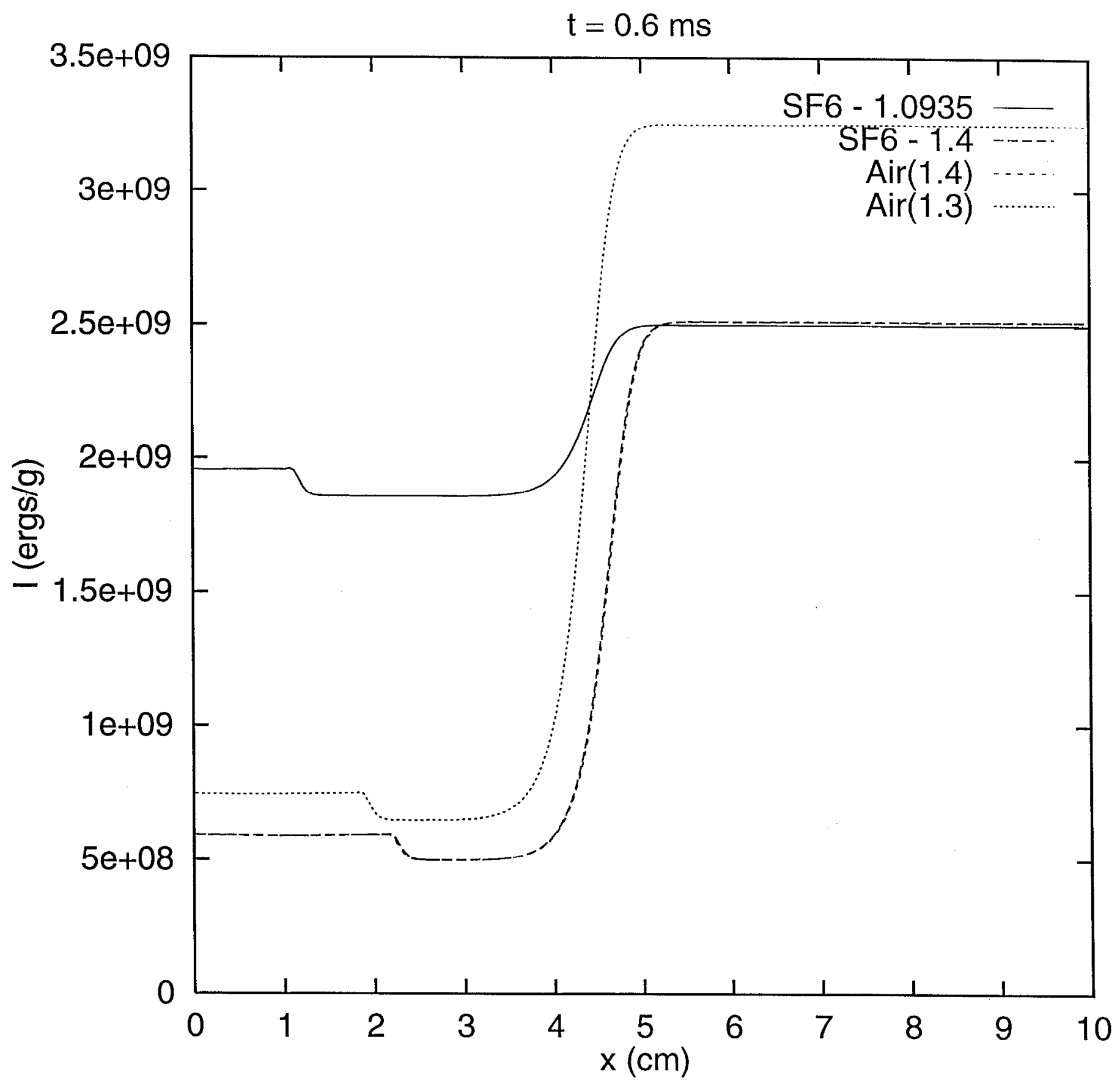

Figure 11: Internal energy as a function of $x$ at $t=0.6 \mathrm{~ms}$ for all four cases. The two dashed lines coincide in this plot. 


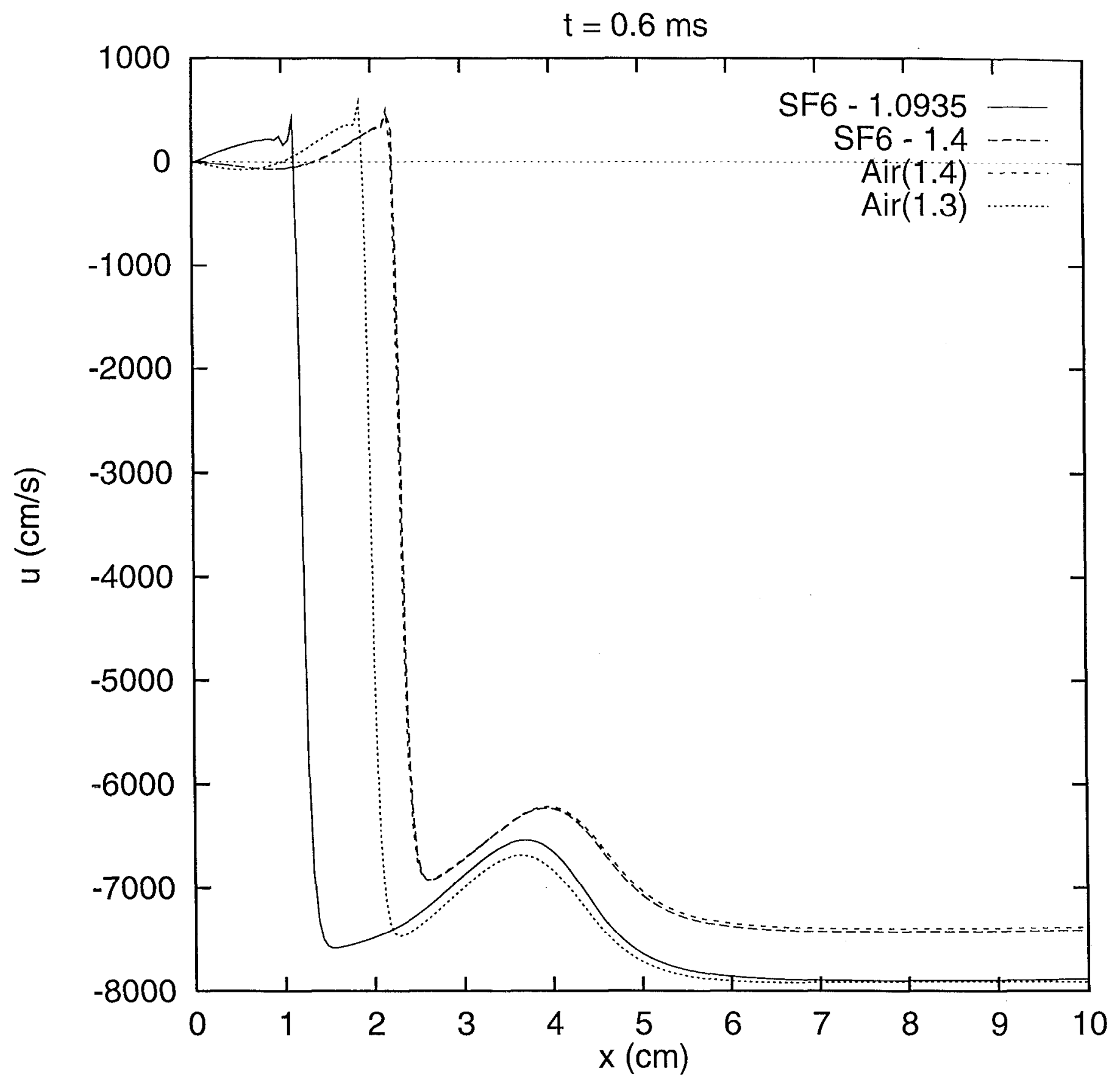

Figure 12: Velocity as a function of $x$ at $t=0.6 \mathrm{~ms}$ for all four cases. The two dashed lines nearly coincide in this plot. 
